ANNALES

POLONICI MATHEMATICI

$85.1(2005)$

\title{
Enclosing solutions of second order equations
}

\author{
by Gerd Herzog and Roland Lemmert (Karlsruhe)
}

\begin{abstract}
We apply Max Müller's Theorem to second order equations $u^{\prime \prime}=f\left(t, u, u^{\prime}\right)$ to obtain solutions between given functions $v, w$.

1. Introduction. Let $I \subseteq \mathbb{R}$ be an interval, and let $v, w \in C^{2}(I, \mathbb{R})$ with $v(t) \leq w(t)(t \in I)$. Let

$$
S:=\{(t, x): t \in I, v(t) \leq x \leq w(t)\}
$$
\end{abstract}

and let $f: S \times \mathbb{R} \rightarrow \mathbb{R}$ be continuous. Consider the second order equation

$$
u^{\prime \prime}(t)=f\left(t, u(t), u^{\prime}(t)\right) .
$$

We are interested in the existence of a solution $u: I \rightarrow \mathbb{R}$ of (1). Then in particular graph $u \subseteq S$, that is, $v(t) \leq u(t) \leq w(t)$ on $I$.

Let $k, l: I \rightarrow \mathbb{R}$ be continuous and such that the equation

$$
h^{\prime \prime}(t)+k(t)\left|h^{\prime}(t)\right|+l(t) h(t)=0
$$

has a positive solution $h: I \rightarrow(0, \infty)$. Under these assumptions we prove

THEOREM 1. If

(i) $|f(t, x, p)-f(t, x, q)| \leq k(t)|p-q|((t, x) \in S, p, q \in \mathbb{R})$,

(ii) $v^{\prime \prime}(t)+l(t) v(t) \leq f\left(t, x, v^{\prime}(t)\right)+l(t) x((t, x) \in S)$,

(iii) $w^{\prime \prime}(t)+l(t) w(t) \geq f\left(t, x, w^{\prime}(t)\right)+l(t) x((t, x) \in S)$,

then (1) has a solution $u: I \rightarrow \mathbb{R}$.

REMARKs. If $f(t, x, p)=f(t, x)$ and $k(t)=0$, conditions (i)-(iii) reduce to

$$
v^{\prime \prime}(t)+l(t) v(t) \leq f(t, x)+l(t) x \leq w^{\prime \prime}(t)+l(t) w(t) \quad((t, x) \in S),
$$

which are satisfied for example if $f(t, x)+l(t) x$ is increasing in $x \in[v(t), w(t)]$ for each $t \in I$ and if

$$
v^{\prime \prime}(t) \leq f(t, v(t)), \quad w^{\prime \prime}(t) \geq f(t, w(t)) \quad(t \in I) .
$$

This case is covered by the result in [2].

2000 Mathematics Subject Classification: 34A40, 34C11.

Key words and phrases: second order equations, Max Müller's Theorem, rotationally symmetric solutions. 
Schrader [6] proved the existence of a solution $u$ of (1) between $v$ and $w$ under the assumptions that

$$
v^{\prime \prime}(t) \leq f\left(t, v(t), v^{\prime}(t)\right), \quad w^{\prime \prime}(t) \geq f\left(t, w(t), w^{\prime}(t)\right) \quad(t \in I),
$$

that $f$ is continuous on $I \times \mathbb{R}^{2}$, that all solutions of initial value problems for equation (1) exist on $I$, and that Dirichlet boundary value problems for (1) on compact subintervals of $I$ have at most one solution.

Moreover, as described in [2] the differential inequalities above should not be mixed up with upper and lower solutions of boundary value problems in the sense of Nagumo [4], where the inequalities are in opposite direction. The following trivial example $(f=0)$ shows most clearly the difference from the method of upper and lower solutions for boundary value problems:

For $I=[a, b]$ there is an affine function between $v \leq w$ if $v^{\prime \prime} \leq 0$ and $w^{\prime \prime} \geq 0$, but in general it is not possible to prescribe boundary values between $v(a) \leq w(a)$ and $v(b) \leq w(b)$.

On the other hand, Rachůnková [5] proves the existence of solutions of (1) satisfying various boundary conditions, which satisfy $v\left(t_{u}\right) \leq u\left(t_{u}\right) \leq w\left(t_{u}\right)$ for some $t_{u} \in I$.

2. Max Müller's Theorem. Let $\mathbb{R}^{2}$ be ordered by the natural cone $K=\{(x, y): x \geq 0, y \geq 0\}$. To prove Theorem 1 we make use of the following two-dimensional version of Max Müller's Theorem [3] (see also $[7])$ :

Let $\xi=\left(\xi_{1}, \xi_{2}\right), \eta=\left(\eta_{1}, \eta_{2}\right) \in C^{1}\left([a, b], \mathbb{R}^{2}\right)$ with $\xi(t) \leq \eta(t)$ on $[a, b]$, and let

$$
D:=\left\{(t, x, y) \in[a, b] \times \mathbb{R}^{2}: \xi(t) \leq(x, y) \leq \eta(t)\right\} .
$$

Let $F=\left(F_{1}, F_{2}\right): D \rightarrow \mathbb{R}^{2}$ be continuous such that for $(t, x, y) \in D$,

$$
\begin{array}{ll}
\xi_{1}^{\prime}(t) \leq F_{1}\left(t, \xi_{1}(t), y\right), & \xi_{2}^{\prime}(t) \leq F_{2}\left(t, x, \xi_{2}(t)\right), \\
\eta_{1}^{\prime}(t) \geq F_{1}\left(t, \eta_{1}(t), y\right), & \eta_{2}^{\prime}(t) \geq F_{2}\left(t, x, \eta_{2}(t)\right),
\end{array}
$$

and let $\xi(a) \leq\left(x_{0}, y_{0}\right) \leq \eta(a)$. Then the initial value problem

$$
(x, y)^{\prime}(t)=F(t, x(t), y(t)), \quad(x(a), y(a))=\left(x_{0}, y_{0}\right)
$$

has a solution $(x, y):[a, b] \rightarrow \mathbb{R}^{2}$; in particular $\operatorname{graph}(x, y) \subseteq D$, that is, $\xi(t) \leq(x(t), y(t)) \leq \eta(t)$ on $[a, b]$.

3. Proof of Theorem 1. First, we prove the assertion for any compact interval $[a, b] \subseteq I$. Let $h:[a, b] \rightarrow \mathbb{R}$ be a positive solution of (2), and let

$$
\bar{v}:=v / h, \quad \bar{w}:=w / h .
$$

Fix $t_{0} \in[a, b]$ such that

$$
\bar{w}\left(t_{0}\right)-\bar{v}\left(t_{0}\right)=\min \{\bar{w}(t)-\bar{v}(t): t \in[a, b]\},
$$


and note that

$$
\begin{aligned}
t_{0}=a & \Rightarrow \bar{v}^{\prime}\left(t_{0}\right) \leq \bar{w}^{\prime}\left(t_{0}\right), \\
t_{0} \in(a, b) & \Rightarrow \bar{v}^{\prime}\left(t_{0}\right)=\bar{w}^{\prime}\left(t_{0}\right), \\
t_{0}=b & \Rightarrow \bar{v}^{\prime}\left(t_{0}\right) \geq \bar{w}^{\prime}\left(t_{0}\right) .
\end{aligned}
$$

We first consider the case $t_{0} \in[a, b)$, and prove

$$
\bar{v}^{\prime}(t) \leq \bar{w}^{\prime}(t)\left(t \in\left[t_{0}, b\right]\right) .
$$

By using (2) we have

$$
\bar{v}^{\prime \prime}=\frac{v^{\prime \prime}}{h}+l \bar{v}+k \frac{\left|h^{\prime}\right|}{h} \bar{v}-\frac{2 h^{\prime}}{h} \bar{v}^{\prime},
$$

and by (ii) with $x=v(t)$,

$$
\begin{aligned}
\bar{v}^{\prime \prime} & \leq \frac{1}{h} f\left(t, v, v^{\prime}\right)+l \bar{v}+k \frac{\left|h^{\prime}\right|}{h} \bar{v}-\frac{2 h^{\prime}}{h} \bar{v}^{\prime} \\
& =\frac{1}{h} f\left(t, v, h^{\prime} \bar{v}+h \bar{v}^{\prime}\right)+l \bar{v}+k \frac{\left|h^{\prime}\right|}{h} \bar{v}-\frac{2 h^{\prime}}{h} \bar{v}^{\prime} .
\end{aligned}
$$

Analogously, from

$$
\bar{w}^{\prime \prime}=\frac{w^{\prime \prime}}{h}+l \bar{w}+k \frac{\left|h^{\prime}\right|}{h} \bar{w}-\frac{2 h^{\prime}}{h} \bar{w}^{\prime}
$$

we get by (iii) and again for $x=v(t)$,

$$
\begin{aligned}
\bar{w}^{\prime \prime} & \geq \frac{1}{h} f\left(t, v, w^{\prime}\right)+l \bar{v}+k \frac{\left|h^{\prime}\right|}{h} \bar{w}-\frac{2 h^{\prime}}{h} \bar{w}^{\prime} \\
& =\frac{1}{h} f\left(t, v, h^{\prime} \bar{w}+h \bar{w}^{\prime}\right)+l \bar{v}+k \frac{\left|h^{\prime}\right|}{h} \bar{w}-\frac{2 h^{\prime}}{h} \bar{w}^{\prime} .
\end{aligned}
$$

Let $G:[a, b] \times \mathbb{R}^{2} \rightarrow \mathbb{R}^{2}$ be defined by

$$
G(t, x, y)=\left(\begin{array}{c}
y \\
\frac{1}{h(t)} f\left(t, v(t), h^{\prime}(t) x+h(t) y\right)+l(t) \bar{v}(t)+k(t) \frac{\left|h^{\prime}(t)\right|}{h(t)} x-\frac{2 h^{\prime}(t)}{h(t)} y
\end{array}\right) .
$$

By (i), the functions

$$
p \mapsto f(t, z, p)+k(t) p, \quad p \mapsto f(t, z,-p)+k(t) p
$$

are increasing on $\mathbb{R}$, so the second coordinate of $G$ is increasing in $x$, and the first coordinate is increasing in $y$. Hence $G$ is quasimonotone increasing in $(x, y)$ with respect to the cone $K=\{(x, y): x \geq 0, y \geq 0\}$ (cf. [8]). Moreover $G$ is continuous and Lipschitz continuous in $(x, y)$. From the estimates for $\bar{v}^{\prime \prime}, \bar{w}^{\prime \prime}$ above we obtain

$$
\left(\begin{array}{c}
\bar{v} \\
\bar{v}^{\prime}
\end{array}\right)^{\prime}-G\left(t, \bar{v}(t), \bar{v}^{\prime}(t)\right) \leq\left(\begin{array}{l}
0 \\
0
\end{array}\right) \leq\left(\begin{array}{c}
\bar{w} \\
\bar{w}^{\prime}
\end{array}\right)^{\prime}-G\left(t, \bar{w}(t), \bar{w}^{\prime}(t)\right)
$$


for $t \in\left[t_{0}, b\right]$. Together with $\left(\bar{v}\left(t_{0}\right), \bar{v}^{\prime}\left(t_{0}\right)\right) \leq\left(\bar{w}\left(t_{0}\right), \bar{w}^{\prime}\left(t_{0}\right)\right)$, a classical result on differential inequalities (see [8, Satz 2]) implies

$$
\left(\bar{v}(t), \bar{v}^{\prime}(t)\right) \leq\left(\bar{w}(t), \bar{w}^{\prime}(t)\right) \quad\left(t \in\left[t_{0}, b\right]\right) .
$$

Next, consider equation (1). The transformation $\bar{u}:=u / h$ leads to

$$
\begin{aligned}
\bar{u}^{\prime \prime} & =\frac{u^{\prime \prime}}{h}-\frac{h^{\prime \prime}}{h} \bar{u}-\frac{2 h^{\prime}}{h} \bar{u}^{\prime} \\
& =\frac{1}{h} f\left(t, u, u^{\prime}\right)+\left(l+k \frac{\left|h^{\prime}\right|}{h}\right) \bar{u}-\frac{2 h^{\prime}}{h} \bar{u}^{\prime} \\
& =\frac{1}{h} f\left(t, h \bar{u}, h^{\prime} \bar{u}+h \bar{u}^{\prime}\right)+\left(l+k \frac{\left|h^{\prime}\right|}{h}\right) \bar{u}-\frac{2 h^{\prime}}{h} \bar{u}^{\prime} .
\end{aligned}
$$

We fix $c_{0} \in\left[\bar{v}\left(t_{0}\right), \bar{w}\left(t_{0}\right)\right]$ and $c_{1} \in\left[\bar{v}^{\prime}\left(t_{0}\right), \bar{w}^{\prime}\left(t_{0}\right)\right]$, and consider the initial value problem

$$
\left(x^{\prime}(t), y^{\prime}(t)\right)=F(t, x(t), y(t)), \quad\left(x\left(t_{0}\right), y\left(t_{0}\right)\right)=\left(c_{0}, c_{1}\right),
$$

with

$$
D:=\left\{(t, x, y): t \in\left[t_{0}, b\right],\left(\bar{v}(t), \bar{v}^{\prime}(t)\right) \leq(x, y) \leq\left(\bar{w}(t), \bar{w}^{\prime}(t)\right)\right\},
$$

and $F=\left(F_{1}, F_{2}\right): D \rightarrow \mathbb{R}^{2}$ defined by

$$
F(t, x, y)=\left(\begin{array}{c}
y \\
\frac{1}{h(t)} f\left(t, h(t) x, h^{\prime}(t) x+h(t) y\right)+\left(l(t)+k(t) \frac{\left|h^{\prime}(t)\right|}{h(t)}\right) x-\frac{2 h^{\prime}(t)}{h(t)} y
\end{array}\right) .
$$

Note that if $(x, y):\left[t_{0}, b\right] \rightarrow \mathbb{R}^{2}$ is a solution of $(3)$, then $u(t)=h(t) x(t)$ is a solution of $(1)$ on $\left[t_{0}, b\right]$.

For $(t, x, y) \in D$ we obviously have

$$
\bar{v}^{\prime}(t) \leq F_{1}(t, \bar{v}(t), y)=y,
$$

and

$$
\left(\bar{v}^{\prime}\right)^{\prime}(t) \leq F_{2}\left(t, x, \bar{v}^{\prime}(t)\right)
$$

follows from the following inequalities (note that $(t, h(t) x) \in S$ ): From (i) we obtain

$$
f\left(t, h x, h^{\prime} \bar{v}+h \bar{v}^{\prime}\right)-f\left(t, h x, h^{\prime} x+h \bar{v}^{\prime}\right) \leq k\left|h^{\prime}\right|(x-\bar{v}) .
$$

Hence

$$
\begin{aligned}
F_{2}\left(t, x, \bar{v}^{\prime}\right) & =\frac{1}{h} f\left(t, h x, h^{\prime} x+h \bar{v}^{\prime}\right)+\left(l+k \frac{\left|h^{\prime}\right|}{h}\right) x-\frac{2 h^{\prime}}{h} \bar{v}^{\prime} \\
& \geq \frac{1}{h} f\left(t, h x, h^{\prime} \bar{v}+h \bar{v}^{\prime}\right)-k \frac{\left|h^{\prime}\right|}{h}(x-\bar{v})+\left(l+k \frac{\left|h^{\prime}\right|}{h}\right) x-\frac{2 h^{\prime}}{h} \bar{v}^{\prime}
\end{aligned}
$$




$$
\begin{aligned}
& =\frac{1}{h} f\left(t, h x, v^{\prime}\right)+l x+k \frac{\left|h^{\prime}\right|}{h} \bar{v}-\frac{2 h^{\prime}}{h} \bar{v}^{\prime} \\
& =\frac{1}{h} f\left(t, h x, v^{\prime}\right)+\frac{l}{h}(h x)+k \frac{\left|h^{\prime}\right|}{h} \bar{v}-\frac{2 h^{\prime}}{h} \bar{v}^{\prime},
\end{aligned}
$$

which by (ii) is

$$
\geq \frac{v^{\prime \prime}}{h}+l \bar{v}+k \frac{\left|h^{\prime}\right|}{h} \bar{v}-\frac{2 h^{\prime}}{h} \bar{v}^{\prime}=\bar{v}^{\prime \prime} .
$$

Analogously

$$
\bar{w}^{\prime}(t) \geq F_{1}(t, \bar{w}(t), y)=y,
$$

and

$$
\left(\bar{w}^{\prime}\right)^{\prime}(t) \geq F_{2}\left(t, x, \bar{w}^{\prime}(t)\right) .
$$

According to Max Müller's Theorem we have a solution of (3), hence a solution $u:\left[t_{0}, b\right] \rightarrow \mathbb{R}$ of the initial value problem

$$
u^{\prime \prime}(t)=f\left(t, u(t), u^{\prime}(t)\right), \quad u\left(t_{0}\right)=h\left(t_{0}\right) c_{0}, u^{\prime}\left(t_{0}\right)=h^{\prime}\left(t_{0}\right) c_{0}+h\left(t_{0}\right) c_{1},
$$

on $\left[t_{0}, b\right]$.

In case $t_{0} \in(a, b]$ we consider the initial value problem (4) to the left, i.e., for any $\varphi:[a, b] \rightarrow \mathbb{R}$ we set

$$
\varphi_{-}(t)=\varphi(a+b-t) \quad(t \in[a, b]),
$$

and define $S_{-}$and $f_{-}: S_{-} \times \mathbb{R} \rightarrow \mathbb{R}$ by

$$
S_{-}=\left\{(t, x): t \in[a, b], v_{-}(t) \leq x \leq w_{-}(t)\right\}
$$

and

$$
f_{-}(t, x, p)=f(a+b-t, x,-p) .
$$

Now, (2) and (i)-(iii) hold for $h, k, l, v, w$, and $S, f$ replaced by $h_{-}, k_{-}, l_{-}$, $v_{-}, w_{-}$, and $S_{-}, f_{-}$, respectively. Since also

$$
\bar{v}^{\prime}\left(t_{0}\right) \geq \bar{w}^{\prime}\left(t_{0}\right) \Rightarrow\left(\bar{v}_{-}\right)^{\prime}\left(a+b-t_{0}\right) \leq\left(\bar{w}_{-}\right)^{\prime}\left(a+b-t_{0}\right),
$$

the first part of our proof, where $t_{0}$ is replaced by $a+b-t_{0}$, gives a solution $u_{-}:\left[a+b-t_{0}, b\right] \rightarrow \mathbb{R}$ of

$$
\begin{gathered}
\left(u_{-}\right)^{\prime \prime}(t)=f_{-}\left(t, u_{-}(t),\left(u_{-}\right)^{\prime}(t)\right), \\
u_{-}\left(a+b-t_{0}\right)=h\left(t_{0}\right) c_{0}, \quad\left(u_{-}\right)^{\prime}\left(a+b-t_{0}\right)=-h^{\prime}\left(t_{0}\right) c_{0}-h\left(t_{0}\right) c_{1},
\end{gathered}
$$

and $u=\left(u_{-}\right)_{-}$solves $(4)$ on $\left[a, t_{0}\right]$.

If, in case $t_{0} \in(a, b)$, we choose $c_{0} \in\left[\bar{v}\left(t_{0}\right), \bar{w}\left(t_{0}\right)\right], c_{1}=\bar{v}^{\prime}\left(t_{0}\right)=\bar{w}^{\prime}\left(t_{0}\right)$, we may put together the solutions obtained by the above procedure to get a solution of (4) on $[a, b]$, which a fortiori satisfies $v \leq u \leq w$ on $[a, b]$.

To prove the theorem on the given interval $I$, which we may assume to be noncompact, we choose an increasing sequence $\left(I_{n}\right)_{n=1}^{\infty}$ of compact intervals 
such that

$$
I=\bigcup_{n=1}^{\infty} I_{n} .
$$

If $I$ contains one of its boundary points, it belongs to some $I_{n_{0}}$, and we assume $n_{0}=1$ without loss of generality. Next, for each $n$ we choose a solution $u_{n}: I_{n} \rightarrow \mathbb{R}$ of (1) such that

$$
v(t) \leq u_{n}(t) \leq w(t) \quad\left(t \in I_{n}\right)
$$

We fix $n \in \mathbb{N}$ and consider $u_{m}, m \geq n$. Then $\left|u_{m}^{\prime \prime}(t)\right| \leq \max \left\{|f(\tau, x, 0)|: \tau \in I_{n}, v(\tau) \leq x \leq w(\tau)\right\}+k(t)\left|u_{m}^{\prime}(t)\right|\left(t \in I_{n}\right)$, from which we get (by [1, Chapter XII, Lemma 5.1]) a constant $L_{n} \geq 0$ such that

$$
\left|u_{m}^{\prime}(t)\right| \leq L_{n} \quad\left(m \geq n, t \in I_{n}\right) .
$$

By a standard diagonal procedure and Ascoli-Arzelà's Theorem we get a subsequence $\left(u_{n_{k}}\right)$ which (together with the first and second derivatives) is locally uniformly convergent on $I$. Its limit is then a solution $u: I \rightarrow \mathbb{R}$ of (1) such that $v(t) \leq u(t) \leq w(t)$ on $I$.

4. Examples. Let $g:[0, \infty) \times \mathbb{R}^{2} \rightarrow \mathbb{R}$ be continuous and bounded $(\alpha \leq g \leq \beta)$, and Lipschitz continuous in its third variable. Let $\|\cdot\|$ denote Euclid's norm on $\mathbb{R}^{n}, n \geq 2$. The classical Ansatz for rotationally symmetric solutions of

$$
\Delta z(\xi)=g(\|\xi\|, z(\xi),\|(\operatorname{grad} z)(\xi)\|) \quad\left(\xi \in \mathbb{R}^{n}\right)
$$

is the transformation $u(\|\xi\|)=z(\xi)$, leading to the singular problem

$$
u^{\prime \prime}(t)=g\left(t, u(t),\left|u^{\prime}(t)\right|\right)-\frac{n-1}{t} u^{\prime}(t) \quad(t \in(0, \infty)) .
$$

We may choose $l(t)=0$ and $k(t)=k_{0}+(n-1) / t$ with $k_{0}$ any Lipschitz constant of $p \mapsto g(t, x, p)$. Then $h(t)=1$ solves $(2)$. Fix $c \in \mathbb{R}$ and consider

$$
v(t)=\frac{\alpha}{2 n} t^{2}+c, \quad w(t)=\frac{\beta}{2 n} t^{2}+c .
$$

Then

$$
\begin{aligned}
v^{\prime \prime}(t) & =\frac{\alpha}{n}=\alpha-\frac{\alpha}{n}(n-1) \\
& \leq g\left(t, x, v^{\prime}(t)\right)-\frac{n-1}{t} v^{\prime}(t) \quad(x \in \mathbb{R}, t \in(0, \infty)),
\end{aligned}
$$

and

$$
\begin{aligned}
w^{\prime \prime}(t) & =\frac{\beta}{n}=\beta-\frac{\beta}{n}(n-1) \\
& \geq g\left(t, x, w^{\prime}(t)\right)-\frac{n-1}{t} w^{\prime}(t) \quad(x \in \mathbb{R}, t \in(0, \infty)) .
\end{aligned}
$$


By Theorem 1 there is a solution $u:(0, \infty) \rightarrow \mathbb{R}$ of $(6)$ with $v(t) \leq u(t) \leq$ $w(t)(t \in(0, \infty))$. In particular the extension $u(0)=c$ leads to $u^{\prime}(0)=0$. By elementary calculus, $u \in C^{2}([0, \infty), \mathbb{R})$. Therefore $z(\xi):=u(\|\xi\|)$ is in $C^{2}\left(\mathbb{R}^{n}, \mathbb{R}\right)$, and is a symmetric solution of equation (5) such that

$$
\frac{\alpha}{n}\|\xi\|^{2}+c \leq z(\xi) \leq \frac{\beta}{n}\|\xi\|^{2}+c \quad\left(\xi \in \mathbb{R}^{n}\right) .
$$

REMARK. In general there is no harmonic function between $v \leq w$ if $v$ is superharmonic and $w$ is subharmonic [2].

In our second example we consider the case $f(t, x, p)=f(t, x), k(t)=0$, and constant functions $v(t)=m, w(t)=M(t \in I)$. Then conditions (i)-(iii) reduce to

$$
l(t) m \leq f(t, x)+l(t) x \leq l(t) M \quad(t \in I, m \leq x \leq M) .
$$

If $f$ is of the form $f(t, x)=l(t) g(t, x)$ and $l(t) \geq 0$ these inequalities hold if

$$
m \leq g(t, x)+x \leq M \quad(t \in I, m \leq x \leq M) .
$$

Consider for example $I=(0,1)$,

$$
h(t)=t(1-t), \quad l(t)=\frac{2}{t(1-t)},
$$

for which $(2)$ holds, and $g(t, x)=\cos (t x)-x$. By Theorem 1 there is a solution $u:(0,1) \rightarrow \mathbb{R}$ of

$$
u^{\prime \prime}(t)=\frac{2(\cos (t u(t))-u(t))}{t(1-t)}
$$

with $-1 \leq u(t) \leq 1(t \in(0,1))$.

\section{References}

[1] P. Hartman, Ordinary Differential Equations, Wiley, New York, 1964.

[2] R. Lemmert, Über gewöhnliche Differentialungleichungen zweiter Ordnung, Proc. Amer. Math. Soc. 83 (1981), 720-724.

[3] M. Müller, Über das Fundamentaltheorem in der Theorie der gewöhnlichen Differentialgleichungen, Math. Z. 26 (1927), 619-645.

[4] N. Nagumo, Über die Differentialgleichung $y^{\prime \prime}=f\left(x, y, y^{\prime}\right)$, Proc. Phys.-Math. Soc. Japan III. Ser. 19 (1937), 861-866.

[5] I. Rachůnková, Upper and lower solutions satisfying the inverse inequality, Ann. Polon. Math. 65 (1997), 235-244.

[6] K. W. Schrader, Differential inequalities for second and third order equations, J. Differential Equations 25 (1977), 203-215.

[7] R. Uhl, An extension of Max Müller's theorem to differential equations in ordered Banach spaces, Funkcial. Ekvac. 39 (1996), 203-216. 
[8] P. Volkmann, Gewöhnliche Differentialungleichungen mit quasimonoton wachsenden Funktionen in topologischen Vektorräumen, Math. Z. 127 (1972), 157-164.

Mathematisches Institut I

Universität Karlsruhe

D-76128 Karlsruhe, Germany

E-mail: Gerd.Herzog@math.uni-karlsruhe.de

Roland.Lemmert@math.uni-karlsruhe.de

Reçu par la Rédaction le 7.10.2004

Révisé le 27.1.2005 